I consider this an excellent successor to Dr Pratt's book. Inevitably it is larger in order to maintain the high standard of being a comprehensive source of reference. Because it presents a clear and logical account of a complex subject it is tremendously helpful. It is essentially a book for neurologists and for them I strongly recommend it.

SARAH Bundey

Orthopaedic Problems in Inherited Skeletal Disorders

By Frank Horan and Peter Beighton. (Pp 142; 98 figures. $£ 20 \cdot 00, \mathrm{DM}$ 85.) Berlin: Springer-Verlag. 1982.

This little book is well-produced with excellent illustrations. It is easy to read and (important) light to carry around.

The authors deal first in a very simple way with genetic principles; the second chapter describes in a general way the investigation and management of bone dysplasias, with one page allotted to antenatal diagnosis. Chromosomal disorders and polygenic inheritance with the common multifactorial disorders are not dealt with. Chapter three contains a full list of the international nomenclature, devised by the European Society of Pediatric Radiologists in Paris in 1969 and subsequently brought up to date. However, the classification adopted by the authors in discussing separate topics adopts the more usual and useful radiological divisions of predominantly disordered epiphyses, metaphyses, with or without vertebral involvement, decreased or increased bone density, etc.

This little book is best considered as a mini atlas and an introduction to the subject of skeletal dysplasias, rather than a technical guide to the postgraduate orthopaedic, plastic, or neuro-surgeon.

It is perhaps a little unrealistic to look for a volume containing the indications for and timing of surgical intervention with pre- and postoperative management in these rare and difficult disorders. It is only relatively recently that a few orthopaedic surgeons have turned their attention to this subject and there is as yet little material on which to base definitive decisions.

Ruth WyNNe-Davies

\section{Clinical Genetics: Problems in Diagnosis and Counseling}

Edited by A M Willey, T P Carter, S Kelly, and I H Porter. (Pp xi +276 ; figures + tables.) New York, London: Academic Press. 1982.
This useful book reports the proceedings of the Twelfth Annual Birth Defects Symposium held in Albany in the autumn of 1981. The distinguished authors, all experts in their field, provide readable accounts of the current state of their art and this volume should prove valuable to anyone attempting to keep abreast of contemporary developments in the rapidly expanding world of medical genetics.

The book opens with an authoritative discussion of the vitamin supplementation studies on the prevention of neural tube defects, followed by a clear and concise overview of the 'new genetics' and its application in the antenatal diagnosis of the haemoglobinopathies. There are three papers with a biochemical flavour dealing with the difficult issues of duration of therapy in phenylketonuria (unresolved) and its management in pregnancy (which should commence before conception), plus the technical aspects of lysosomal enzyme assay and complementation studies with special reference to propionicacidaemia.

There then follow four papers of cytogenetic interest. The first indicates that the risk of 'severe anomaly' in a fetus with an apparently balanced de novo structural rearrangement is unlikely to be great (three out of 41) and may not differ significantly from that for the general population. The need for more data is freely admitted. There is a helpful review of mosaicism and pseudomosaicism in antenatal cytogenetic diagnosis although the problem of trisomy 20 mosaicism remains. A study of "who gets amniocentesis' is well worth reading, as is an excellent discussion of recurrence risks in Down's syndrome, which offers useful guidance on how to cater for paternal age, previous non-disjunction, and parental remarriage.

Chapters of general interest include a comprehensive account of the overgrowth syndromes, a review of empirical risk data in psychiatric disorders, and a brief but fascinating glimpse into the mind of Francis Galton, whose interests ranged from spectacles for divers to the statistical inefficacy of prayer (those who were prayed for most, such as royalty, tended to die youngest). It is a mixed comfort to note that Dr Opitz estimates that he can identify a cause and recurrence risk in only $20 \%$ of cases with an unknown syndrome. What hope is there for the rest of us lesser mortals? More depressing are the papers on cystic fibrosis, which reveal that despite considerable progress, reliable antenatal diagnosis is still some way off.

In presenting the proceedings of any symposium the editors and publishers face a difficult task in maintaining literary and technical quality without sacrificing speed of production. In this volume they have largely succeeded. The text is of a high standard, 
the illustrations and tables are clear, and the index is comprehensive. It is, however, a little unfortunate that some 15 months should elapse between the symposium and its publication on this side of the Atlantic. This minor criticism apart, this book can be strongly recommended to anyone actively involved in the practice of clinical genetics.

I D Young

\section{Sister Chromatid Exchange}

Progress and Topics in Cytogenetics. Vol 2. Edited by Avery A Sandberg. (Pp xv + 706; figures + tables. $£ 66 \cdot 50$, DM 293.) New York: Alan R Liss. 1982.

Suitably stained chromosome preparations made from cells grown in the presence of bromodeoxyuridine, which is incorporated into newly synthesised DNA in place of thymidine, reveal 'harlequin' chromosomes which display with diagrammatic clarity the difference between chromatids with bromodeoxyuridine substituted and unsubstituted DNA strands. This beautiful demonstration of the operation of semiconservative replication can also be utilised to show, with great precision, exchanges between sister chromatids. A technique of such relative simplicity which comes close to demonstrating molecular events in the structures in which it occurs is a pleasure to use. It is not surprising that workers in laboratories around the world have taken to this technique not only to study cell kinetics but also to investigate the significance of chromosome exchange phenomena in relation to damage and repair of the genetic machinery and the induction of neoplasia.

In this volume Dr Sandberg has gathered some 30 contributions on sister chromatid exchange, 31 if you count one paper on the induction of micronuclei. The contributions are loosely grouped under general headings. 'History and Methodology' is appropriately opened by Zakharov whose introduction in 1972 of bromodeoxyuridine techniques marked the transformation of the study of SCE from the rather limited observations of the behaviour of ring chromosomes (initiated by McClintock in 1938) and Taylor's now classical autoradiographic studies using ${ }^{3} \mathrm{H}$-thymidine, to the much easier, clearer staining techniques currently available. There follows a 'recipe book' review of staining methods, a paper on the use of SCEs to assess the persistence of DNA lesions, and a paper on the induction of micronuclei.

The second section which consists of five papers and is entitled 'Possible Mechanisms of SCE $\stackrel{\mathbb{P}}{?}$ Formation' illustrates well the fascinating and simultaneously frustrating aspect of SCE study. Model building is fun and various models are reviewed which fit more or less well with presently available evidence, but it seems likely that we must await a better knowledge of the molecular events involved before we can properly understand either the cause or the mechanism of the SCE phenomenon.

Most of the rest of the book has its eye firmly fixed on the goal of providing a means for monitoring the mutagenic and carcinogenic effects of environmental agents. A section on 'SCE in Cells and Tissues of Plants, Animals and Humans' attempts to establish baseline frequencies according to species, tissue, and age with due account of the effect of the experimental procedures in SCE induction. SCEs in meiotic cells also receive attention and comparisons are made with meiotic crossing-over.

There are a number of papers reporting on SCE frequency encountered in human disease states with emphasis on those associated with neoplasia or predisposition to neoplasia. Separate sections take the effect of physical and biological agents, of mutagens and carcinogens, of drugs and the value of SCE analysis in Public Health monitoring as their theme.

The papers in this book succeed in covering the main preoccupations of those using this technique and highlight many of the problems and pitfalls of interpretation. They convey well the dilemma of having a technique which clearly demonstrates an effect of DNA damaging agents on the chromosome which is compatible with continued cellular replication but which has so far resisted attempts to correlate it with known mechanisms of DNA repair. This is probably a reflection of the wide spectrum of DNA damage induced by different agents and the multiplicity of repair systems available to the cell, but is a disappointment to those searching for a reliable indicator of carcinogenic potential.

There is little that is new in this book. The papers are essentially of a review type and, inevitably in a collection of presentations by many different people dealing with one observed chromosomal activity, there is much repetition which a single author would be able to avoid, but the book undoubtedly provides a helpful review and bibliography of work and thought in this area at a time appropriate for appraisal of the usefulness of the techniques involved. There is an index too. However, the considerable cost of the book may be a disincentive to many who may wish to use it.

Michael FAed 